\title{
Multiple level spondylodiscitis with presacral abscess in spinal brucellosis: a rare presentation
}

\author{
Lobo Manuel Alexander ${ }^{1 *}$, SachinAdukia ${ }^{2}$, JigneshPrajapati ${ }^{2}$ \\ ${ }^{I}$ Dept of Neurology, Mazumdar Shaw Medical Center, NH Health City, 258/A, Bommasandra Industrial Area, Hosur road, \\ Bangalore, -560099 Karnataka, India \\ ${ }^{2}$ MD Medicine, Postgraduate Student, Dept of Neurology, Mazumdar Shaw Medical Center, NH Health City, 258/A, \\ Bommasandra Industrial Area, Hosur road, Bangalore, -560099 Karnataka, India \\ *Corresponding author E-mail:lobo13alex@gmail.com
}

\begin{abstract}
Importance: We report an unusual presentation of spinal brucellosis involving multilevel spondylodiscitis, prevertebral sacral abscess and isolation of Brucella using a low-yield test like blood culture. Timely identification helped in dispensing the appropriate antibiotic regimen.

Observation:Detailed history against an endemic background may give clues to diagnosis of brucellosis. A middle aged farmer from rural India with exposure to cattle excreta presented with severe lower backache and asymmetric paraparesis. Examination revealed proximal lower limb weakness, sensory deficits over L1-L2 dermatomes, sensory level at L1 vertebral level and lumbosacral spinal tenderness with paravertebral muscle spasms. MRI spine with contrast showed multi-level infective spondylodiscitis with a prevertebral sacral abscess. Blood culture helped isolate Brucella melitensis after 8 days. Following this the antibiotic regimen was modified to a twelve month triple therapy with rifampicin, doxycycline and double strength cotrimoxazole.

Conclusions and Relevance: Longstanding backache with fever in endemic areas should trigger a differential diagnosis of spinal brucellosis. Though newer diagnostic techniques like ELISA, MRI and agglutination tests are being increasingly used, isolation of Brucella on culture media is still a vital investigation. Diagnosis of brucellosis is vital owing to its potential treatability.
\end{abstract}

Keywords: Brucella Melitensis;Blood Culture; MRI; Multi-Level Spondylodiscitis; Prevertebral Sacral Abscess

\section{Introduction}

Spinal brucellosis is defined as involvement of the vertebral column, interspinal spaces, and/or paraspinal areas. It may present as radiculoneuritis, myelitis, spondylodiscitis, demyelinating neuropathy and epidural abscess.Lesions occur anywhere along the spine but a lumbar region is the commonest site, and rarest is sacral $(0.3 \%)$;incidence of multiple site involvement is $9-30$ $\%($ Alp\&Doganay 2008).Frequency of spondylodiscitis increases with aging (Tur et al. 2004)

\section{Case Report}

A 56-year-old male farmer presented with low-grade fever with chills, insidious onset progressive lumbalgia with radicular pain in lower limbs, asymmetrical paraparesis and reduced sensation over lower back since 9 weeks. He was exposed to cattle excreta while walking barefoot. Clinical examination revealed asymmetrical proximal lower limb weakness (left $3+/ 5$, right $4 / 5$ ). Paravertebral muscle spasm and moderate tenderness in the L1 area and L5-S1 area was recorded along with hypoesthesia in the left L1-L2 dermatomes and sensory level at L1 vertebral level.

MRI spine with contrast showed T2 hyper intensities involving the T12-L2 intervertebral discs, adjoining portions of T11 and T12 vertebral body, and L5-S1 intervertebral disc. Post contrast enhancement of L1, L5 vertebral bodies was seen. A well-defined T2/STIR hyperintense, enhancing abscess in L5-S1 prevertebral region was present. These findings suggested multilevel infective spondylodiscitis and presacral abscess (Figure 1). 

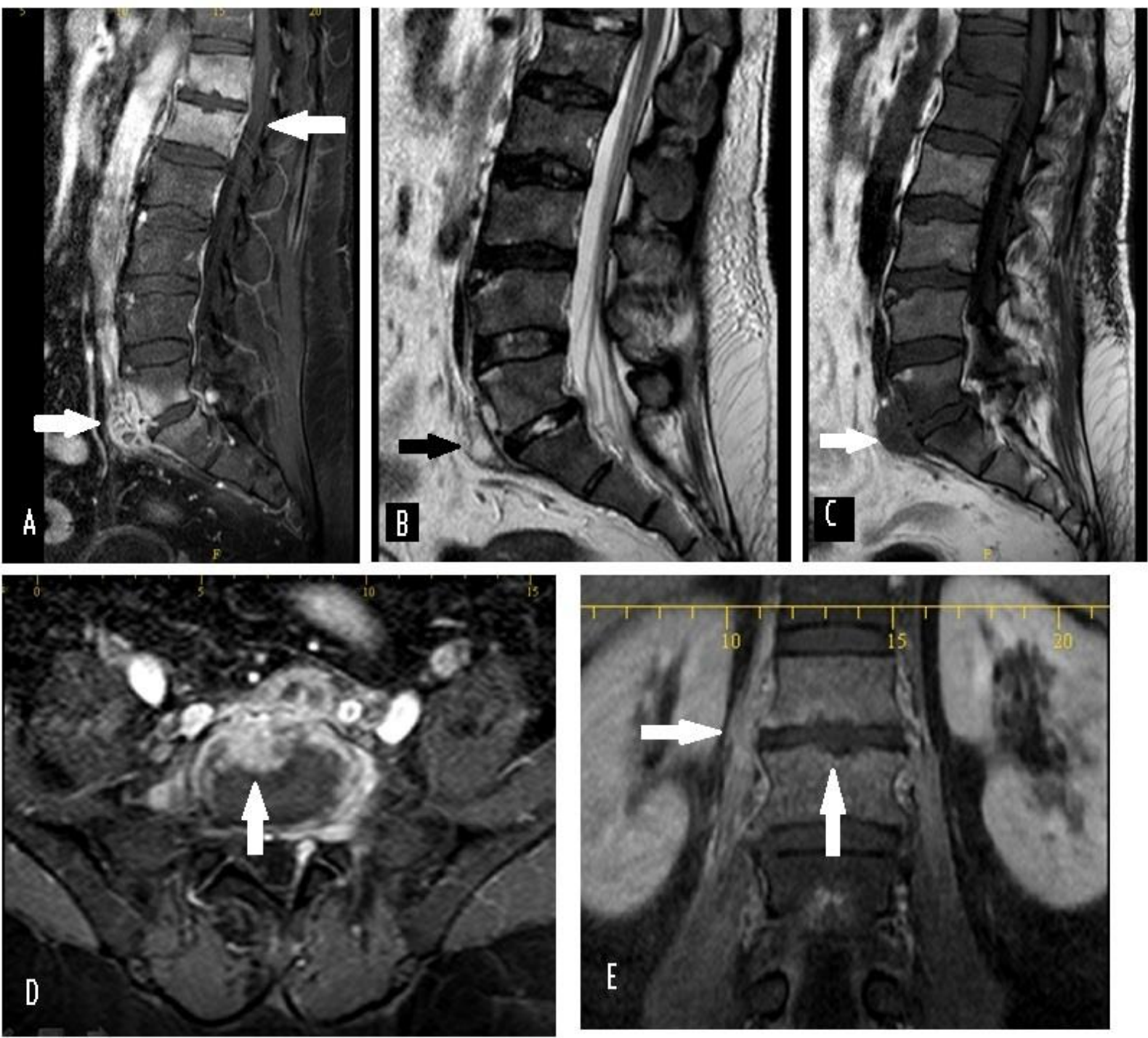

Fig. 1:MRI Lumbosacral Spine Showing Spondylodiscitis, Paravertebral Contrast Enhancement and Presacral Abscess on Different Sections.

Legend to figure 1: A, MRI Lumbosacral spine showing contrast uptake at T12-L1 level (white arrow- right side) and at L5-S1 level with presacral collection (white arrow- left side); $\mathrm{B}$, presacral collection on $\mathrm{T} 2$ imaging (black arrow); C, presacral collection on $\mathrm{T} 1$ imaging (white arrow); $\mathrm{D}$, post-contrast imaging transverse section at L1 level showing spondylodiscitis (white arrow); E, Coronal section on post contrast imaging showing paravertebral enhancement and Schmorl's nodes (Vertical arrow).

CSF analysis was deferred due to multiple level involvement in lumbar region. Standard tube agglutination showed insignificant titre of antibodies to Brucella. Tests for tuberculosis yielded negative results. Empirical intravenous coverage, including ceftriaxone, metronidazole and gentamicin with oral doxycycline was started. Anti-tuberculous drugs were added considering endemicity of tuberculosis in India. Since pyogenic abscess was considered, steroids were not initiated. After eight days, blood culture grew Brucella melitensis. Now, treatment was revised to a twelve month triple therapy with rifampicin, doxycycline and double strength cotrimoxazole.

\section{Discussion}

Early in the course of spinal brucellosis, osteoporosis of affected vertebral body and erosion of the anterior-superior endplate occurs followed by narrowing of joint space. Focal anterior or diffuse disc collapse is seen. Posterior elements are rarely involved. Dis- tinctive MR features include moderately abnormal paraspinal soft tissue without abscess formation, intact vertebral architecture despite diffuse vertebral osteomyelitis and lack of gibbus deformity (Kim \& Cho 2008). Table 1 shows differentiating MRI features between brucellosis and tuberculosis (Beeching\& Corbel 2015).

Table 1: Difference between Radiological Features of Spinal Brucellosis and Spinal Tuberculosis

\begin{tabular}{|c|c|c|}
\hline & Brucellosis & Tuberculosis \\
\hline Site & Lumbar and others & Dorsolumbar \\
\hline Vertebrae & $\begin{array}{l}\text { Multiple or contigu- } \\
\text { ous }\end{array}$ & Contiguous \\
\hline Discitis & Late & Early \\
\hline Body & Intact till late & Morphology lost early \\
\hline Canalcompression & Rare & Common \\
\hline Epiphysitis & Anterosuperior & $\begin{array}{l}\text { General: upper and lower } \\
\text { disc region, cen- } \\
\text { tral,subperiosteal }\end{array}$ \\
\hline Osteophyte & Anterolateral & Uncommon \\
\hline Deformity & $\begin{array}{l}\text { Wedging uncom- } \\
\text { mon }\end{array}$ & $\begin{array}{l}\text { Anterior wedging with gib- } \\
\text { bus }\end{array}$ \\
\hline Recovery & $\begin{array}{l}\text { Sclerosis of whole } \\
\text { vertebral body }\end{array}$ & Variable \\
\hline $\begin{array}{l}\text { Paravertebral } \\
\text { abscess }\end{array}$ & $\begin{array}{l}\text { Small, well local- } \\
\text { ized }\end{array}$ & Common and discrete \\
\hline Psoas abscess & Rare & More likely \\
\hline
\end{tabular}

Slow growing bacteria and intermittent bacteremia decrease the yield of blood culture. Notwithstanding, growth of Brucella organisms in culture of blood, bone marrow or involved tissue is diagnostic (Kim \& Cho 2008). Differential diagnosis includes tubercu- 
lous spondylitis, salmonella spondylitis, other pyogenic spondylitis, disc herniation, and vertebral metastases (Nas et al. 2001).

WHO recommends first line combination therapy for spinal brucellosis with tetracycline/doxycycline for 6 weeks with an aminoglycoside (preferably streptomycin for 7-10 days). Combination of rifampin and doxycycline for 8 weeks is an alternative therapy. Fluoroquinolones have good penetration and achieve good tissue concentrations, which may lead to better outcomes. Fluoroquinolone and rifampicin combination is as effective as doxycycline and rifampin. However, specific guidelines regarding antibiotic regimens and duration of treatment for neuro brucellosis are still lacking. Surgical intervention is indicated in spinal instability, cord compression, radiculopathy, cauda equine syndrome, and severe weakness of the muscles due to extradural inflammatory mass. Epidural and paravertebral abscesses may be given a trial of antibiotics. In case of no response or neurological deterioration percutaneous drainage of epidural abscess can be performed (Alp \&Doganay 2008).

Our case was unique due to multilevel spondylodiscitis, prevertebral sacral abscess and isolation of Brucella from blood culture. Involvement of posterior vertebral elements, as seen in this case, is uncommon. To conclude, MRI finding of spondylodiscitis in endemic areas should prompt a differential diagnosis and evaluation for brucellosis. This is vital considering the potential treatability of the condition.

\section{References}

[1] Alp E, Doganay M. Current therapeutic strategy in spinal brucellosis. Int $\mathrm{Jr}$ of Infectious Dis. 2008 Nov 30; 12(6):573-7. https://doi.org/10.1016/j.ijid.2008.03.014.

[2] Tur BS, Suldur N, Ataman S, Ozturk EA, Bingol A, Atay MB. Brucellar spondylitis: a rare cause of spinal cord compression. Spinal Cord.2004 May 1;42(5):321 4.https://doi.org/10.1038/sj.sc.3101571.

[3] Kim DH, Cho YD. A case of spondylodiscitis with spinal epidural abscess due to Brucella. Journal of Korean Neurosurgical Society. 2008 Jan 1; 43(1):37-40. https://doi.org/10.3340/jkns.2008.43.1.37.

[4] Beeching NJ, Corbel MJ. Brucellosis. In: Loscalzo J, Kasper D, Hauser S, Fauci A, Jameson J, Longo D, eds. Harrison's Principles of Internal Medicine 19th Edition. New York: McGraw Hill; 2015. p. $1066-1069$

[5] Nas K, Gür A, Kemaloglu MS, Geyik MF, Cevik R, Büke Y, Ceviz A, Sarac AJ, Aksu Y. Management of spinal brucellosis and outcome of rehabilitation. Spinal Cord. 2001 Apr 1; 39(4):2237.https://doi.org/10.1038/sj.sc.3101145. 